\title{
The Airy 1 process is not the limit of the largest eigenvalue in GOE matrix diffusion
}

\author{
Folkmar Bornemann*, Patrik L. Ferrari†, Michael Prähofer ${ }^{\ddagger}$
}

June 20, 2008

\begin{abstract}
Using a systematic approach to evaluate Fredholm determinants numerically, we provide convincing evidence that the Airy ${ }_{1}$-process, arising as a limit law in stochastic surface growth, is not the limit law for the evolution of the largest eigenvalue in GOE matrix diffusion.
\end{abstract}

\section{Introduction}

One of the unsolved problems in random matrix theory is to understand the law for the largest eigenvalue in GOE matrix diffusion. Let $M(t)$ be a matrixvalued stationary process on real symmetric matrices of size $N \times N$ satisfying: (i) the one-time distribution of $M(t)$ is given by the Gaussian Orthogonal Ensemble (GOE), (ii) the (independent) entries of $M(t)$ are independent stationary Ornstein-Uhlenbeck processes in time. The corresponding process for the ordered eigenvalues $\lambda_{N, j}(t), j=1, \ldots, N$ is Dyson's Brownian motion with $\beta=1$. The stationary distribution of the largest eigenvalue, $\lambda_{N, N}(t)$, can be expressed fairly explicit, and its limiting distribution, under proper rescaling as $N \rightarrow \infty$, is the GOE Tracy-Widom distribution [16]. However, no simple expression for the joint distribution of the largest eigenvalue at two different times is known. To be specific, one can ask for the covariance of the largest eigenvalue $\operatorname{Cov}\left(\lambda_{N, N}(t), \lambda_{N, N}(0)\right)$ and its asymptotic behavior as $N \rightarrow \infty$.

For the related case of GUE matrix diffusion, i.e., when $M(t)$ is a hermitian matrix and the stationary distribution is given by the Gaussian Unitary

*Technische Universität München, e-mail: bornemann@ma.tum.de

${ }^{\dagger}$ Weierstrass Institute, WIAS Berlin, e-mail: ferrari@wias-berlin.de

${ }^{\ddagger}$ Technische Universität München, e-mail: praehofer@ma.tum.de 
Ensemble (GUE), the corresponding question is answered. In this case the law for the eigenvalues is given by Dyson's Brownian motion with $\beta=2$ and the limiting process of the properly rescaled largest eigenvalue is the Airy process. This process first arose in the context of one-dimensional stochastic surface growth with curved macroscopic shape [14] for the so-called polynuclear growth (PNG) model.

This raised the question, whether the limit process of the largest eigenvalue in GOE matrix diffusion can also be obtained from a growth process. A strong candidate was one-dimensional growth starting from a flat substrate, since in this case the limiting one-point distribution is the same as for GOE matrix diffusion [13]. This correspondence was partially extended to a multilayer version of flat growth with non-intersecting height lines. It was shown in [8] that the point process of the multilayer at a fixed position and the point process of the GOE ensemble at the edge of the spectrum have the same asymptotic law.

The analogue of the Airy process for flat growth was discovered by Sasamoto in a growth model related to PNG [15]. Since its defining kernel at equal times is, in a certain sense, the square root of the standard Airy kernel [10], it was baptized the "Airy 1 process". Accordingly, for better distinction, we call the standard Airy process "Airy 2 process" in the rest of the paper.

In [4] two conjectures have been formulated. The first predicted that the Airy $_{1}$ process is also the limit process for the PNG model with flat initial conditions, which subsequently has been proven in [6]. The second claimed

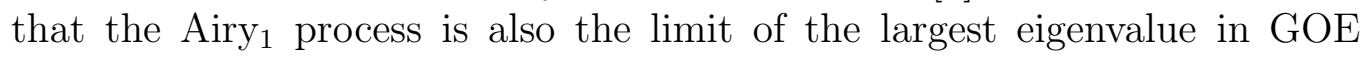
matrix diffusion ( $\beta=1$ Dyson's Brownian motion).

In this paper we show that the second conjecture does not hold. To

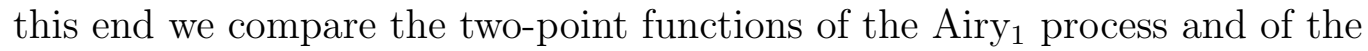
largest eigenvalue in GOE matrix diffusion for different matrix sizes.

The joint distribution functions for the Airy processes are given in terms of Fredholm determinants of integral operators. To evaluate these Fredholm determinants we employ a numerical scheme, recently developed by one of the authors [2], which in itself is of general interest. For matrix diffusion we use straightforward Monte-Carlo simulations on large matrices.

The comparison shows that the correlation function for GOE matrix diffusion differs, in the limit of large matrices, from the one for Airy ${ }_{1}$. In contrast, in the case of GUE matrix diffusion, the corresponding numerical calculations perfectly illustrate the known convergence to the Airy 2 process. 


\section{Polynuclear growth model}

In this section we present the polynuclear growth model in $1+1$ dimensions and give known results relevant for the discussion. We refer to the original papers for more details.

\section{The model and its multilayer extension}

We briefly define the polynuclear growth (PNG) model on a one-dimensional substrate. At time $t$, the surface is described by an integer-valued height function $x \mapsto h(x, t) \in \mathbb{Z}, x \in \mathbb{R}, t \in \mathbb{R}_{+}$, with steps of size 1 , which is taken to be upper semicontinuous, i.e., $\lim _{x \rightarrow x_{0}} h(x, t) \leq h\left(x_{0}, t\right)$ for all $x_{0}, t$. Thus the surface consists of up-steps $(\ulcorner)$ and down-steps $(\beth)$. The dynamics of these steps has a deterministic and a stochastic part:

(i) up- (down-) steps move to the left (right) with unit speed. When a down-step and an up-step collide they simply disappear.

(ii) pairs of up- and down- steps at the same point (spikes) are produced by random nucleation events with some given intensity. The up- and down-steps of the spikes then spread out with unit speed according to (i).

The multilayer extension of the PNG model [14] is the following. Instead of a single height function $h(x, t)$ we have a set of height functions $\left\{h_{\ell}(x, t), \ell \leq 0\right\}$, with the initial condition $h_{\ell}(x, 0)=\ell$, for all $x \in \mathbb{R}$. The dynamics of $h_{0}(x, t)$ is the same as for the original $h(x, t)$. For the remaining lines (i) applies as for $h_{0}(x, t)$. Rule (ii) is modified insofar, that for $h_{\ell}(x, t)$, $\ell \leq-1$, nucleation events are not produced at random, but whenever there is a collision of a pair of steps in level $\ell+1$ at $(x, t)$, a spike is produced in level $\ell$ at $(x, t)$. By construction the lines do not intersect and one associates an (extended) point process $\eta$ on $\mathbb{R} \times \mathbb{Z}$, by

$$
\eta(x, j)= \begin{cases}1, & h_{\ell}(x, t)=j \text { for some } \ell \leq 0 \\ 0, & \text { otherwise }\end{cases}
$$

\section{The PNG droplet}

Consider a flat initial substrate $h(x, 0)=0, x \in \mathbb{R}$. The PNG droplet is obtained when the nucleations form a Poisson point process in spacetime with intensity $\rho(x, t)=2$ for $|x| \leq t$ and $\rho(x, t)=0$ otherwise. For 
large growth time $t$, the interface has the shape of a droplet, namely the deterministic limit,

$$
h_{\mathrm{ma}}(\xi):=\lim _{t \rightarrow \infty} t^{-1} h(\xi t, t)=2 \sqrt{1-\xi^{2}} \mathbb{1}_{(|\xi| \leq 1)} .
$$

The fluctuations of the height function grow as $t^{1 / 3}$ and the correlation length as $t^{2 / 3}$. Therefore, the edge scaling of the (multilayer) height functions around the origin, $x=0$, is given by

$$
h_{\ell}^{\text {droplet }}(u, t):=\frac{h_{\ell}\left(u t^{2 / 3}\right)-t h_{\mathrm{ma}}\left(u t^{-1 / 3}\right)}{t^{1 / 3}} .
$$

For the PNG droplet, the point process associated to the multilayer is determinantal. Moreover, rescaled as in (2.3), it converges in the large $t$ limit to the Airy field [14], defined by the n-point correlation functions

$$
\rho^{(n)}\left(u_{1}, s_{1} ; \ldots ; u_{n}, s_{n}\right)=\operatorname{det}\left(K_{\mathcal{A}_{2}}\left(u_{i}, s_{i} ; u_{j}, s_{j}\right)\right)_{1 \leq i, j \leq n},
$$

where

$$
K_{\mathcal{A}_{2}}\left(u, s ; u^{\prime}, s^{\prime}\right)=\left\{\begin{array}{cl}
\int_{0}^{\infty} \mathrm{d} \lambda e^{\left(u^{\prime}-u\right) \lambda} \operatorname{Ai}(s+\lambda) \operatorname{Ai}\left(s^{\prime}+\lambda\right), & u^{\prime} \leq u, \\
-\int_{-\infty}^{0} \mathrm{~d} \lambda e^{\left(u^{\prime}-u\right) \lambda} \operatorname{Ai}(s+\lambda) \operatorname{Ai}\left(s^{\prime}+\lambda\right), & u^{\prime}>u .
\end{array}\right.
$$

Denote by $\mathcal{A}_{2}(u)$ the highest point of the Airy field at position $u$. It can be seen as a process $u \mapsto \mathcal{A}_{2}(u)$ and it is called the Airy $y_{2}$ process. The convergence of the extended point process to the Airy field implies in particular that [14]

$$
\lim _{t \rightarrow \infty} h_{0}^{\text {droplet }}(u, t)=\mathcal{A}_{2}(u) .
$$

The joint distributions of the Airy, process are given by Fredholm determinants: for any given $u_{1}<u_{2}<\ldots<u_{m}$, and $s_{1}, \ldots, s_{m} \in \mathbb{R}$,

$$
\mathbb{P}\left(\bigcap_{k=1}^{m}\left\{\mathcal{A}_{2}\left(u_{k}\right) \leq s_{k}\right\}\right)=\operatorname{det}\left(\mathbb{1}-\chi_{s} K_{\mathcal{A}_{2}} \chi_{s}\right)_{L^{2}\left(\left\{u_{1}, \ldots, u_{m}\right\} \times \mathbb{R}\right)}
$$

where $\chi_{s}\left(u_{k}, x\right)=\mathbb{1}_{\left(x>s_{k}\right)}$. This expression allows to determine some properties of the covariance

$$
g_{2}(u):=\operatorname{Cov}\left(\mathcal{A}_{2}(u), \mathcal{A}_{2}(0)\right),
$$

namely

$$
g_{2}(0)=\operatorname{Var}\left(\mathcal{A}_{2}(0)\right)=0.81320 \ldots, \quad g_{2}^{\prime}(0)=-1,
$$


and the asymptotics for large $u[1,17]$,

$$
g_{2}(u)=\frac{1}{u^{2}}+\frac{c}{u^{4}}+\mathcal{O}\left(u^{-6}\right),
$$

with the constant $c=-3.542 \ldots$, evaluated numerically from an explicit expression in terms of the Hastings-McLeod solution of Painlevé II [2].

\section{The flat PNG}

Consider a flat initial substrate $h(x, 0)=0, x \in \mathbb{R}$, and run the PNG dynamics with constant nucleation intensity, say $\rho(x, t)=2$ for all $x \in \mathbb{R}$, $t \geq 0$. Then the limit shape is flat, $h_{\mathrm{ma}}(\xi)=2$. Thus the edge scaling is

$$
h_{\ell}^{\text {flat }}(u, t):=\frac{h_{\ell}\left(u t^{2 / 3}\right)-2 t}{t^{1 / 3}} .
$$

For the flat PNG, the correlation structure of the multilayer version is not known, but a few results are available.

(a) In the large time limit the point process corresponding to (2.11) for a fixed value of $u$ converges to a Pfaffian point process [8] whose $n$-point correlation functions are given by

$$
\rho^{(n)}\left(s_{1}, \ldots, s_{n}\right)=2^{2 n / 3} \operatorname{Pf}\left(G^{\mathrm{GOE}}\left(2^{2 / 3} s_{i} ; 2^{2 / 3} s_{j}\right)\right)_{1 \leq i, j \leq n} .
$$

$G^{\mathrm{GOE}}$ is a $2 \times 2$ matrix kernel (for an explicit expression see, e.g. (2.9) in [8]) and $\mathrm{Pf}$ is the Pfaffian $(\operatorname{Pf}(A)=\sqrt{\operatorname{det}(A)}$ for an antisymmetric matrix $A)$. This kernel also occurs for GOE random matrices in the large matrix limit at the edge of the spectrum.

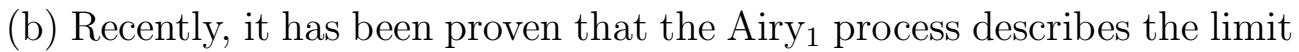
of the top line of the multilayer flat PNG [5], namely

$$
\lim _{t \rightarrow \infty} h_{0}^{\text {flat }}(u, t)=2^{1 / 3} \mathcal{A}_{1}\left(u / 2^{2 / 3}\right) .
$$

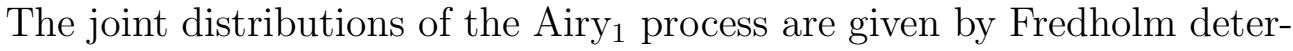
minants: for any given $u_{1}<u_{2}<\ldots<u_{m}$, and $s_{1}, \ldots, s_{m} \in \mathbb{R}$,

$$
\mathbb{P}\left(\bigcap_{k=1}^{m}\left\{\mathcal{A}_{1}\left(u_{k}\right) \leq s_{k}\right\}\right)=\operatorname{det}\left(\mathbb{1}-\chi_{s} K_{\mathcal{A}_{1}} \chi_{s}\right)_{L^{2}\left(\left\{u_{1}, \ldots, u_{m}\right\} \times \mathbb{R}\right)},
$$

where $\chi_{s}\left(u_{k}, x\right)=\mathbb{1}\left(x>s_{k}\right)$, and the kernel $K_{\mathcal{A}_{1}}$ is defined by

$$
\begin{aligned}
K_{\mathcal{A}_{1}}\left(u, s ; u^{\prime}, s^{\prime}\right) & =\operatorname{Ai}\left(s+s^{\prime}+\left(u-u^{\prime}\right)^{2}\right) \exp \left(\left(u^{\prime}-u\right)\left(s+s^{\prime}\right)+\frac{2}{3}\left(u^{\prime}-u\right)^{3}\right) \\
& -\frac{1}{\sqrt{4 \pi\left(u^{\prime}-u\right)}} \exp \left(-\frac{\left(s^{\prime}-s\right)^{2}}{4\left(u^{\prime}-u\right)}\right) \mathbb{1}\left(u^{\prime}>u\right)
\end{aligned}
$$


Some properties of the Airy 1 process like the short and long time behavior of the covariance are known. We refer to the review [9] for details. In particular, the short-time behavior of the covariance of the Airy 1 process,

$$
g_{1}(u):=\operatorname{Cov}\left(\mathcal{A}_{1}(u), \mathcal{A}_{1}(0)\right)
$$

satisfies

$$
g_{1}(0)=\operatorname{Var}\left(\mathcal{A}_{1}(0)\right) \simeq 0.402 \ldots, \quad g_{1}^{\prime}(0)=-1 .
$$

\section{Dyson's Brownian motion}

Dyson's Brownian motion [7] describes the diffusion of $N$ mutually repelling particles with positions $\lambda_{j}(t), j=1, \ldots, N$, at time $t$ on the real line in a harmonic potential,

$$
\mathrm{d} \lambda_{j}(t)=\left(-\gamma \lambda_{j}(t)+\frac{\beta}{2} \sum_{i \neq j} \frac{1}{\lambda_{j}(t)-\lambda_{i}(t)}\right) \mathrm{d} t+\mathrm{d} b_{j}(t), \quad j=1, \ldots, N,
$$

the $b_{j}(t)$ being independent standard Brownian motions with $\operatorname{Var}\left(b_{j}(t)\right)=$ $t$. Let $P(\lambda)$ denote the probability distribution of particle positions $\lambda=$ $\left(\lambda_{1}, \ldots, \lambda_{N}\right)$. It satisfies the diffusion equation

$$
\frac{\partial}{\partial t} P(\lambda)=\sum_{j=1}^{N} \frac{\partial}{\partial \lambda_{j}}\left(\gamma \lambda_{j} P(\lambda)-\frac{\beta}{2} \sum_{i \neq j} \frac{1}{\lambda_{j}-\lambda_{i}} P(\lambda)\right)+\frac{1}{2} \frac{\partial^{2}}{\partial \lambda_{j}^{2}} P(\lambda) .
$$

The stationary distribution is given by

$$
P(\lambda)=\frac{1}{Z}|\Delta(\lambda)|^{\beta} \exp \left(-\gamma \sum_{j=1}^{N} \lambda_{j}^{2}\right)
$$

with $\Delta(\lambda)=\prod_{1 \leq i<j \leq N}\left(\lambda_{j}-\lambda_{i}\right)$, and $Z$ the normalization.

In his original work, Dyson linked the special values $\beta=1,2,4$ to the eigenvalue GOE, GUE and GSE random matrices, respectively. In these cases, $\lambda_{j}(t)$ is the $j$-th smallest eigenvalue of a random matrix $M(t)$ diffusing according to an Ornstein-Uhlenbeck process on real symmetric, hermitian or symplectic matrices, respectively.

We describe the correspondence only in the cases $\beta=1$ (GOE) and $\beta=2$ (GUE). Let $b_{i j}^{\alpha}(t), 1 \leq i, j \leq N, \alpha=1,2$, be independent standard Brownian motions. In the GOE case one sets $b_{i j}(t)=b_{i j}^{1}(t) \in \mathbb{R}$, in the GUE case one sets $b_{i j}(t)=b_{i j}^{1}(t)+\mathrm{i} b_{i j}^{2}(t) \in \mathbb{C}$. Let $B_{i j}(t)=\frac{1}{2}\left(b_{i j}(t)+\right.$ 
$\left.\overline{b_{j i}(t)}\right)$. Now $B(t)$ is a Brownian motion on the space of real symmetric, resp., hermitian matrices, which is invariant with respect to orthogonal, resp. unitary rotations. For GOE the independent entries are $B_{i j}, j \geq i$, while for GUE the independent entries are $B_{i i}$ and $\operatorname{Re}\left(B_{i j}\right), \operatorname{Im}\left(B_{i j}\right), j>i$. These real-valued independent entries perform Brownian motions, with variance $t$ on the diagonal and variance $t / 2$ for the remaining entries. Now let $M(t)$ be the stationary Ornstein-Uhlenbeck process defined by

$$
\mathrm{d} M(t)=-\gamma M(t) \mathrm{d} t+\mathrm{d} B(t) .
$$

The stationary distribution is proportional to $\exp \left(-\gamma \operatorname{Tr}\left(M^{2}\right)\right)$ in both cases, GOE and GUE. By integrating over the angular variables, one gets the stochastic evolution of eigenvalues as in (3.3).

Let us mention here, that the parameter $\gamma$ is in fact irrelevant. Multiplying the eigenvalues by $\sqrt{\gamma}$ and rescaling time by $\gamma^{-1}$ one can always arrange for $\gamma=1$. We kept this parameter throughout the formulas to facilitate comparisons with the literature, where different, sometimes $N$-dependent, conventions for $\gamma$ have been adopted. The most common choice, $\gamma=1$, leads to the standard Hermite kernel with Hermite polynomials orthogonal with respect to the weight $e^{-x^{2}}$.

\section{GUE diffusion and Airy process}

In the case $\beta=2$, the point process associated to the ordered eigenvalues, $\lambda_{j}(t)$ of $M(t)$ is determinantal, defined by the extended Hermite kernel [12]. The edge scaling at the upper edge of the spectrum is given by

$$
\lambda_{N, j}^{\mathrm{GUE}}(u)=\sqrt{2 \gamma} N^{1 / 6}\left(\lambda_{j}\left(u /\left(\gamma N^{1 / 3}\right)\right)-\sqrt{2 N / \gamma}\right) .
$$

Under this rescaling, the kernel of the corresponding point process converges to the Airy kernel $K_{\mathcal{A}_{2}}$ as $N \rightarrow \infty$ [1]. This allows to show the convergence of the rescaled largest eigenvalue, $\lambda_{N, N}^{\mathrm{GUE}}(u)$, to the Airy 2 process,

$$
\lim _{N \rightarrow \infty} \lambda_{N, N}^{\mathrm{GUE}}(u)=\mathcal{A}_{2}(u),
$$

in the sense of convergence of finite-dimensional distributions [11]. The finite$N$ covariance of the largest eigenvalue is denoted by $f_{N}^{\mathrm{GUE}}$,

$$
f_{N}^{\mathrm{GUE}}(u)=\operatorname{Cov}\left(\lambda_{N, N}^{\mathrm{GUE}}(u), \lambda_{N, N}^{\mathrm{GUE}}(0)\right) .
$$

Obviously one expects that $\lim _{N \rightarrow \infty} f_{N}^{\mathrm{GUE}}(u)=g_{2}(u)$. To prove this rigorously, convergence of moments is needed in (3.6), a result which is currently not available. 


\section{GOE diffusion}

For $\beta=1$, the GOE case, explicit expressions for dynamical correlations are not known. Nevertheless one expects an analogous behavior as for GUE diffusion. In the edge scaling of the ordered eigenvalues $\lambda_{j}(t)$ of $M(t)$ in the GOE case, one has two free parameters (time and space scaling). In order to check the hypothesis that the Airy ${ }_{1}$ process describes the evolution of the largest eigenvalue for the GOE case, we choose the free parameters by matching the covariance and its derivative at zero, see (2.17). We obtain

$$
\lambda_{N, j}^{\mathrm{GOE}}(u)=\sqrt{\gamma} N^{1 / 6}\left(\lambda_{j}\left(2 u /\left(\gamma N^{1 / 3}\right)\right)-\sqrt{N / \gamma}\right) .
$$

As anticipated while speaking of the flat PNG, the point process of the edgerescaled GOE eigenvalues at a fixed time (the one associated to $\left\{\lambda_{N, j}^{\mathrm{GOE}}(0), 1 \leq\right.$ $j \leq N\}$ ) converges to a Pfaffian point process with $n$-point correlation given by

$$
\rho^{(n)}\left(s_{1}, \ldots, s_{n}\right)=2^{n} \operatorname{Pf}\left(G^{\mathrm{GOE}}\left(2 s_{i}, 2 s_{j}\right)\right)_{1 \leq i, j \leq n}
$$

in the $N \rightarrow \infty$ limit. We denote by $f_{N}^{\mathrm{GOE}}$ the finite- $N$ covariance of the largest eigenvalue,

$$
f_{N}^{\mathrm{GOE}}(u)=\operatorname{Cov}\left(\lambda_{N, N}^{\mathrm{GOE}}(u), \lambda_{N, N}^{\mathrm{GOE}}(0)\right) .
$$

The scaling in (3.8) is chosen such that $f_{N}^{\mathrm{GOE}^{\prime}}(0)=-1$ and $f_{N}^{\mathrm{GOE}}(0) \rightarrow g_{1}(0)$ as $N \rightarrow \infty$. As in the GUE case one expects the limit $f_{\infty}^{\mathrm{GOE}}(u)=$ $\lim _{N \rightarrow \infty} f_{N}^{\mathrm{GOE}}(u)$ to exist. In the next section we address the question whether $f_{\infty}^{\mathrm{GOE}}(u)$ equals $g_{1}(u)$.

\section{Numerical results}

The Airy ${ }_{1}$ process was regarded as a candidate for the limit of the rescaled largest GOE eigenvalue process $[4,15]$, because of the known properties of these two processes. Both are stationary processes with the same one-point distribution, and the conjectured short time behaviors coincide, too. Furthermore, as explained above, the underlying multiline ensembles have the same limiting single time distribution as point processes on $\mathbb{R}$ (see (2.12) and (3.9) ). The final ingredient for the guess is that the connections carries over the multilines picture in the $\beta=2$ case.

In lack of more analytical input, we looked for an answer to the question, whether the Airy 1 process is the limit of the $\beta=1$ Dyson's Brownian motion by numerical means. We decided to compare the large $N$ limit of (3.10) with the covariance of the Airy 1 process (2.16). The quantities in question are 


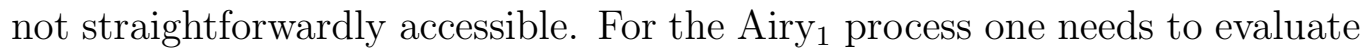
Fredholm determinants. For Dyson's Brownian motion we performed MonteCarlo simulations on the eigenvalues of coupled GOE matrices directly.

\section{Covariances of the Airy $_{1}$ and Airy A processes $^{2}$}

The key point of the numerical computation is the evaluation of the Fredholm determinants of the two-point joint distributions for the Airy and Airy $_{2}$ processes, eqs. (2.7), (2.14). This is explained in details in the recent paper [2]. Let us briefly describe the procedure.

Basic ingredient is a Nyström-type approximation of integral operators by an $n$-point quadrature formula for integrals over the interval $(s, \infty)$ that gives exponential convergence rates for holomorphic integrands. Such a formula can be based on the holomorphic transformation

$$
\phi_{s}:(0,1) \rightarrow(s, \infty), \quad \xi \mapsto s+10 \tan (\pi \xi / 2)
$$

followed by Gauss-Legendre quadrature on the interval $(0,1)$ with weights $w_{j}$ and points $\xi_{j}(j=1, \ldots, n)$. This way we obtain

$$
\int_{s_{k}}^{\infty} f(x) \approx \sum_{j=1}^{n} w_{j} \phi_{s_{k}}^{\prime}\left(\xi_{j}\right) f\left(\phi_{s_{k}}\left(\xi_{j}\right)\right)=\sum_{j=1}^{n} w_{k j} f\left(x_{k j}\right) .
$$

The Fredholm determinants (2.7) and (2.14) are now approximated by the $m n \times m n$-dimensional determinant

$$
\operatorname{det}\left(\mathbb{1}-\chi_{s} K_{\mathcal{A}_{\mu}} \chi_{s}\right)_{L^{2}\left(\left\{u_{1}, \ldots, u_{m}\right\} \times \mathbb{R}\right)} \approx \operatorname{det}\left(\begin{array}{cccc}
\mathbb{1}-A_{11} & A_{12} & \cdots & A_{1 m} \\
A_{21} & \mathbb{1}-A_{22} & \cdots & A_{2 m} \\
\vdots & \vdots & & \vdots \\
A_{m 1} & A_{m 2} & \cdots & \mathbb{1}-A_{m m}
\end{array}\right)
$$

where the submatrices $A_{i j} \in \mathbb{R}^{n \times n}(i, j=1, \ldots, m)$ are defined by

$$
\left(A_{i j}\right)_{p q}=w_{i p}^{1 / 2} K_{\mathcal{A}_{\mu}}\left(u_{i}, x_{i p} ; u_{j}, x_{j q}\right) w_{j q}^{1 / 2} \quad(p, q=1, \ldots, n) .
$$

Theorem 8.1 of [2] shows that the approximation error in (4.1) decays exponentially with $n$, that is, like $O\left(\rho^{-n}\right)$ for some constant $\rho>1$. Thus, doubling $n$ doubles the number of correct digits; a fact on which simple strategies for adaptive error control can be based [3]. Additionally, the level of round-off error can be controlled as described in [2]. It turns out that the two-point $(m=2)$ joint distribution can be calculated to an absolute precision of $10^{-14}$ 
using $n$ quadrature points with $n$ between 20 and 100, depending on the specific values of $u, s_{1}$, and $s_{2}$. The CPU time for a single evaluation of the joint distribution is well below a second (using a $2 \mathrm{GHz} \mathrm{PC}$ ).

The covariances $g_{1}(u)$ and $g_{2}(u)$ were calculated by first truncating the integrals, then integrating by parts (to avoid numerical differentiation), and finally using Clenshaw-Curtis quadrature. A truncation at \pm 10 (except for small $u \leq 0.05$ in the case of $g_{1}$, where larger integration intervals are necessary) and 100 quadrature points in each of the two dimensions are sufficient to secure an absolute precision of $10^{-10}$.

The code for calculating the two-point joint distributions and the covariances $g_{1}$ and $g_{2}$ can be obtained from the first author upon request.

\section{Monte Carlo Simulation of Random Matrices}

To get an estimate for the covariance of the largest eigenvalue of GUE and GOE matrices, we performed straightforward Monte-Carlo simulations. A collection of matrices $C_{k}, k=0, \ldots, K$, independently distributed according to the stationary distribution of (3.4) can be easily produced with standard pseudo random generators, since each $C_{k}$ consists of independently normally distributed entries. Fixing a time step $\Delta t$, it is easy to see that for the stationary process $M(t)$ governed by (3.4) the joint distribution of $(M(k \Delta t))_{0 \leq k \leq K}$ is the same as for the matrices $M_{k}$, defined by

$$
M_{0}=C_{0}, \quad M_{k}=e^{-\gamma \Delta t} M_{k-1}+\sqrt{1-e^{-2 \gamma \Delta t}} C_{k}, \quad 1 \leq k \leq K .
$$

Now, one numerically determines the largest eigenvalues of the $M_{k}$ and rescales according to eqs. (3.5), resp., (3.8). This yields realizations of $\lambda_{N, N}^{\mathrm{GOE}}(u)$ and $\lambda_{N, N}^{\mathrm{GUE}}(u)$ at equidistant times $u$. The empirical auto-covariance of these time series gives an estimate for the covariance functions $f_{N}^{\mathrm{GOE}}(u)$ and $f_{N}^{\mathrm{GUE}}(u)$ at discrete values of $u$. The data presented here are for $N=64$, 128 , and 256 with $\gamma=1 / 2$ and $\Delta t=\frac{1}{2} N^{-1 / 3}$. We chose $K=10^{6}$, and produced up to 100 independent realizations of the time series in each case. This allows to obtain an error estimate for each data point.

\section{Discussion}

In Figure 1 we compare the covariance (2.16) of the Airy 1 process and the one of the largest eigenvalue for GOE matrix diffusion (3.10) for $N=64$ and $N=256$. One clearly sees that they do not agree.

Increasing the matrix dimension does not change sensibly the result; namely, the results for $N=128$ agree to plotting accuracy with the one for $N=256$ in Figure 1 and therefore are not shown. In comparison, in 


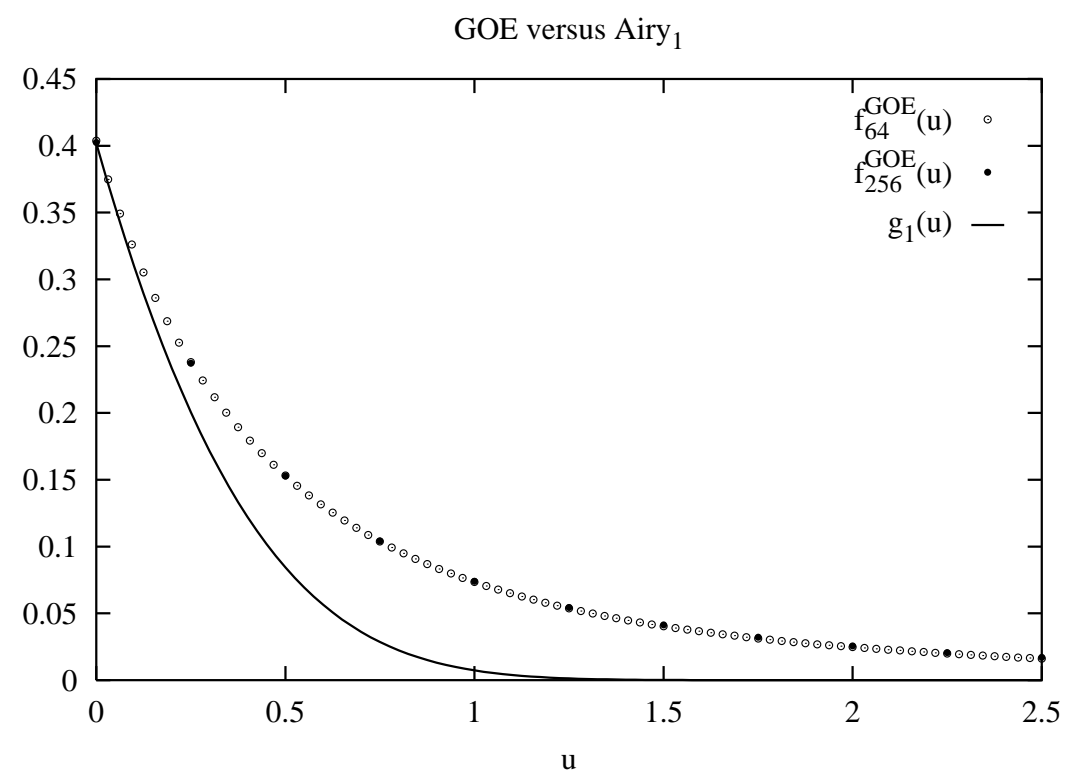

Figure 1: The covariance $g_{1}$ of the Airy 1 process (line) versus the one of the largest eigenvalue for GOE matrix diffusion, $f_{N}^{\mathrm{GOE}}$, for $N=64,256$.

Figure 2 we plot the covariance (2.8) of the Airy 2 process and the one of the largest eigenvalue for GUE matrix diffusion (3.7) for the same matrix dimensions. Here the agreement is already quite good even for relatively small matrix sizes. In both plots the errorbars are of order $10^{-3}$, smaller then the symbols used and therefore omitted.

Concerning the decay of $g_{1}$, it appears to be superexponentially in sharp contrast to the algebraic decay (2.10) of $g_{2}$. After reinspecting the $2 \times 2$ block Fredholm determinant this behavior becomes clear, since one of the off-diagonal blocks is superexponentially small in $u$ for large values of $u$, while the others stay of order one, a fact already noticed by Widom [18].

Finally, in Figure 3, we provide a comparison of the decay of correlation for GOE and GUE matrix diffusion. In a log-log plot we draw $f_{N}^{\mathrm{GOE}}$ and $f_{N}^{\mathrm{GUE}}$ for $N=128$ and $N=256$ with errorbars. For GUE one observes the deviation from the asymptotic behavior $u^{-2}$ for large $u$ due to finite size effects. Remarkably $f_{N}^{\mathrm{GOE}}(u)$ looks very similar to $\frac{1}{2} f_{N}^{\mathrm{GUE}}(2 u)$, indicating that the large $u$ behavior of $f_{\infty}^{\mathrm{GOE}}(u)$ might also be algebraically decaying, in sharp contrast to the superexponential decay of $g_{1}(u)$. Given the small matrix dimensions we used, we can not, however, conclude whether the decay for $f_{\infty}^{\mathrm{GOE}}(u)$ is of order $u^{-2}$ as for GUE or not. 


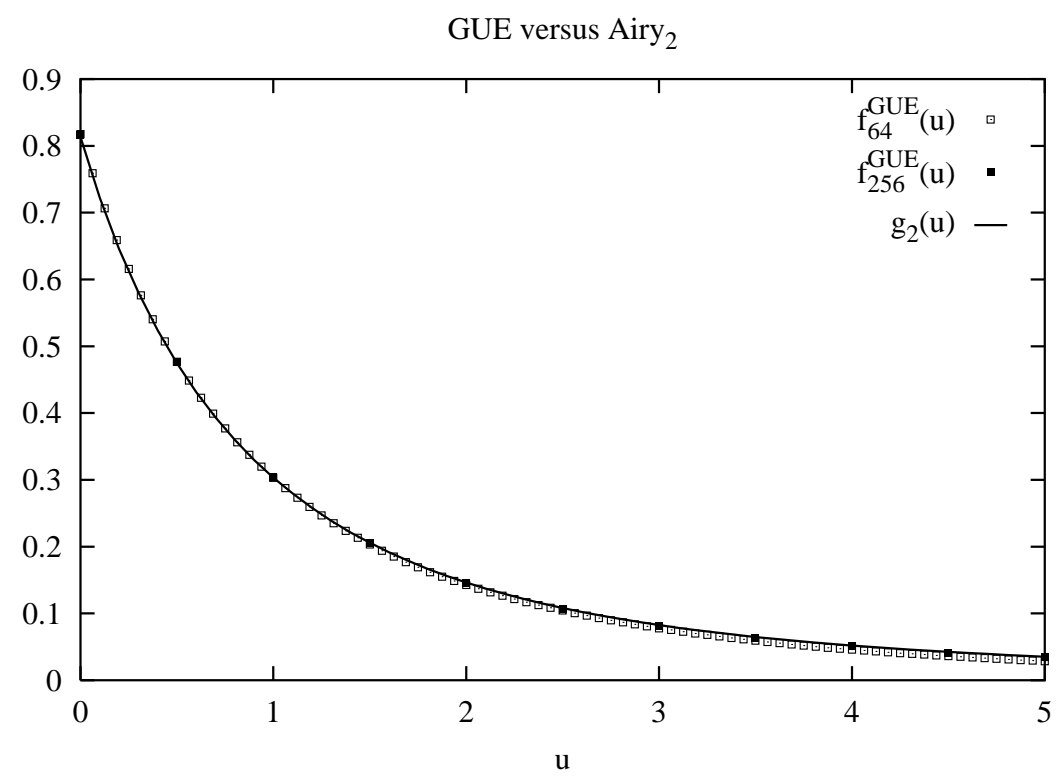

Figure 2: The covariance $g_{2}$ of the Airy 2 process (line) versus the one of the largest eigenvalue for GUE matrix diffusion, $f_{N}^{\mathrm{GUE}}$, for $N=64,256$.

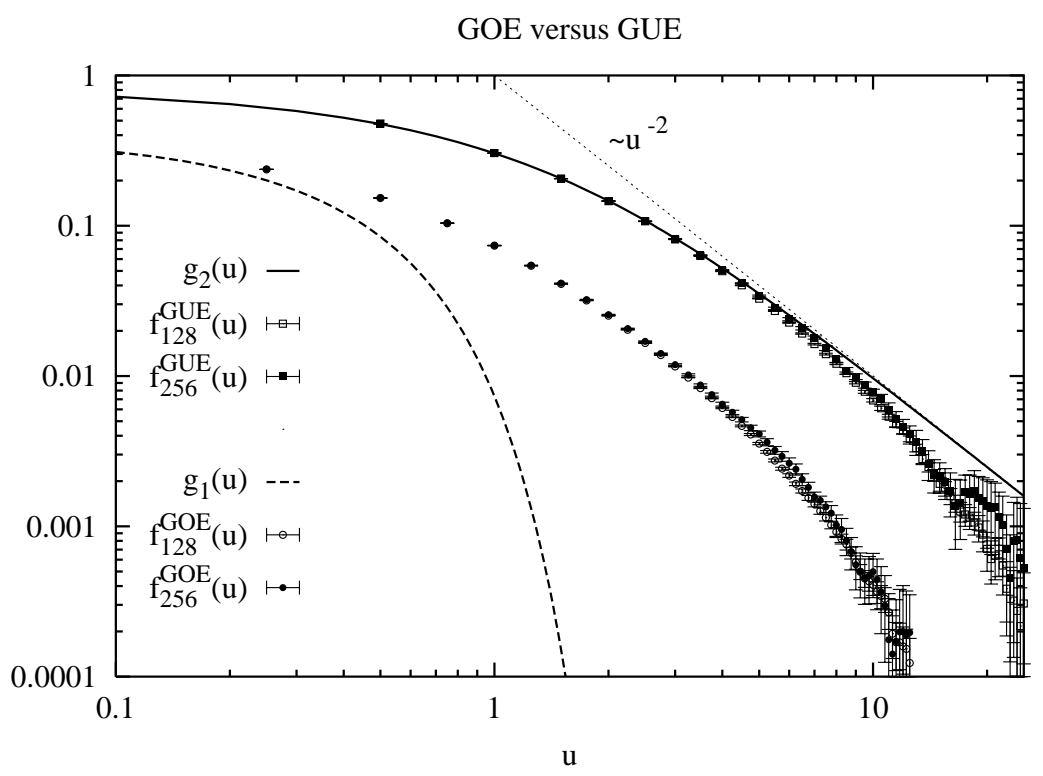

Figure 3: Log-log plot of the rescaled correlation functions for GOE and GUE. 


\section{References}

[1] M. Adler and P. van Moerbeke, PDE's for the joint distribution of the Dyson, Airy and Sine processes, Ann. Probab. 33 (2005), 1326-1361.

[2] F. Bornemann, On the numerical evaluation of Fredholm determinants, arXiv:0804.2543 (2008).

[3] F. Bornemann, D. Laurie, S. Wagon, and J. Waldvogel, The SIAM 100Digit Challenge. A Study in High-Accuracy Numerical Computing, Society of Industrial and Applied Mathematics, Philadelphia, 2004.

[4] A. Borodin, P.L. Ferrari, M. Prähofer, and T. Sasamoto, Fluctuation properties of the TASEP with periodic initial configuration, J. Stat. Phys. 129 (2007), 1055-1080.

[5] A. Borodin, P.L. Ferrari, and T. Sasamoto, Large time asymptotics of growth models on space-like paths II: PNG and parallel TASEP, arXiv:0707.4207; To appear in Comm. Math. Phys. (2007).

[6] A. Borodin, P.L. Ferrari, and T. Sasamoto, Transition between Airy 1 and Airy $_{2}$ processes and TASEP fluctuations, Comm. Pure Appl. Math., online first (2007).

[7] F.J. Dyson, A Brownian-motion model for the eigenvalues of a random matrix, J. Math. Phys. 3 (1962), 1191-1198.

[8] P.L. Ferrari, Polynuclear growth on a flat substrate and edge scaling of GOE eigenvalues, Comm. Math. Phys. 252 (2004), 77-109.

[9] P.L. Ferrari, The universal Airy $1_{1}$ and Airy 2 processes in the Totally Asymmetric Simple Exclusion Process, arXiv:math-ph/0701021. To appear in Contemporary Mathematics, "Proceedings on Integrable Systems, Random Matrices, and Applications. A conference in honor of Percy Deifts 60th birthday" (2007).

[10] P.L. Ferrari and H. Spohn, A determinantal formula for the GOE TracyWidom distribution, J. Phys. A 38 (2005), L557-L561.

[11] K. Johansson, Discrete polynuclear growth and determinantal processes, Comm. Math. Phys. 242 (2003), 277-329.

[12] K. Johansson, Non-intersecting, simple, symmetric random walks and the extended Hahn kernel, Ann. Inst. Fourier, Grenoble 55 (2005), 21292145 . 
[13] M. Prähofer and H. Spohn, Universal distributions for growth processes in $1+1$ dimensions and random matrices, Phys. Rev. Lett. 84 (2000), $4882-4885$.

[14] M. Prähofer and H. Spohn, Scale invariance of the PNG droplet and the Airy process, J. Stat. Phys. 108 (2002), 1071-1106.

[15] T. Sasamoto, Spatial correlations of the $1 D K P Z$ surface on a flat substrate, J. Phys. A 38 (2005), L549-L556.

[16] C.A. Tracy and H. Widom, On orthogonal and symplectic matrix ensembles, Comm. Math. Phys. 177 (1996), 727-754.

[17] H. Widom, On asymptotics for the Airy process, J. Stat. Phys. 115 (2004), 1129-1134.

[18] H. Widom, Private communication, (2008). 